\title{
CONTRAST ENHANCED SUPERPIXEL BASED SEGMENTATION OF RETINAL IMAGES
}

\author{
Abhisha Mano, Assistant Professor, Rajas Institute of Technology for Women,Nagercoil, \\ Email:abhizion1986@gmail.com.
}

ABSTRACT:

Retinal blood vessel segmentation plays a major role in diabetic retinopathy treatment. If it is not diagnosed at the early stage, it will lead to loss of vision. In this paper an efficient segmentation of retinal image using minimum spanning superpixel tree is proposed, to get both global and local information of the retinal images. Contrast limited adaptive histogram equalization (CLAHE) algorithm is applied to images, inorder to achieve local contrast enhancement. The features are extracted from both the illumination and texture layer, which is used for framing superpixel. The blood vessels are segmented using the minimum spanning superpixel tree detector. The results are compared with existing techniques in terms of accuracy, sensitivity, specificity and execution time.It is observed that the proposed method achieves an accuracy of $97.6 \%$ and it completes the execution in $8.50 \mathrm{sec}$.

\section{INTRODUCTION}

Diabetic retinopathy (DR) is a microvascular problem of diabetes and leads to vision loss in the people. If early diagnosis is done, it helps in prevention of vision loss. Dark lesions occur in microaneurysms (MAs) and haemorrhages and bright lesions occur in exudates and cotton wool spots. An automated system is essential for separating healthy and diseased regions in the image which can efficiently decrease the difficulty in large scale screening. Color fundus images are widely used for automatic DR detection. For MA detection many machine learning techniques are used. When the retina is been affected by diabetes, this disease is called Diabetic Retinopathy (DR). Fundus photography gives clear pictures of the internal structures of human eye such as the retina, the optic disc, macula, fovea and blood vessels. Optical Coherent Tomography (OCT) is helpful when the eye diseases are diagnosed at tissue level. Moreover, fundus images are very efficient when the eye diseases are analyzed at retinal level. These images can be found on Standard Diabetic Retinopathy Database (DIARETDB), Structured Analysis of the Retina (STARE) and Digital Retinal Images for Vessel Extraction (DRIVE) etc.

In paper[1], the problem associated with detection of blood vessels in retinal images is discussed. An operator is introduced which depends on the properties of objects to be detected. The objects have both optical and spatial properties. This concept is given for feature extraction. The gray-level profile of each and every blood vessel is equated to a Gaussian function. The matched filter is used to segment blood vessels. The work [2] combines information in all 3 color channels to enhance the segmentation performance. Here effective color fusion scheme is applied. Its performance is evaluated on DRIVE and STARE. The proposed feature fusion with dimensionality reduction by asymmetric PCA visibly enhances the segmentation performance. In paper[3] an automatic extraction of blood vessels from colour retinal images is presented. Line detectors at varying scales are achieved when the length of a basic line detector is varied. The individual line detector has few drawbacks which are eliminated by combining the line responses, by which the final segmentation is done for each retinal image. Here the central reflex vessels are accurately segmented. The measurements of vessel width made on REVIEW dataset is highly accurate. Two segmentation methods are considered in paper[4]. The response obtained from the line detector is thresholded to obtain unsupervised pixel classification. The gray level value of the target pixel is combined with the two orthogonal line detectors, to frame 
a feature vector for supervised classification by means of a support vector machine. In paper [5], accuracy of detecting blood vessels is increased by using matched filter response. Moreover, the threshold value has to be optimized for the matched filter an efficient technique is employed.

The paper [6] uses bagged and boosted decision trees, that depends on a feature vector framed based on gradient vector field, morphological conversion, line strength methods, and Gabor filter outcomes. The method is evaluated on DRIVE and STARE databases. The paper [7] proposes an automated method for identification of blood vessels in color images of the retina. For every image pixel, a feature vector is computed that utilizes properties of scale and orientation selective Gabor filters. The extracted features are then classified using generative Gaussian mixture model and discriminative support vector machines classifiers. Receiver operating characteristic (ROC) curve reached a value of 0.964 in this method. A paper [8] a solution is obtained by the combination of heterogeneous context-aware features along with a discriminative learning technique. A hybrid feature pool with stroke width transform (SWT) and Weber's local descriptors (WLD), and classical local features Gabor responses and vesselness measurements is used. Encode context information by sampling the hybrid features from an orientation invariant local context. Pixel-level vessel segmentation was done and use a random forest to fuse the rich information encoded in the hybrid context-aware features. In paper [9],brain tumor segmentation of MRI images was implemented. In this approach, Kmeans clustering followed by GLCM feature extraction was implemented. In training of ANN, the GSO is used to optimize the weight, which reduces the root mean square error. The initial mask which is obtained from ANN is applied to active contour, whose energy is normalized mean of patches in an image. The accuracy of this is increased by $2 \%$.

This paper [10] entirely focuses on gray scale images and their security by a synchronous transformation and substitution system. In this technique, permutation is achieved by means of arithmetic crossover, multipoint crossovers operator and also substitution by means of combined mutation operators. In this technique key stream is generated by 3-D Compound Sine and ICMIC (CSI) map. Encryption achieved by means of hybrid operators with secret key. In this method, protection of real- time images has been achieved. An algorithm [11] to find out the DNA fragment size from images of DNA molecules. Here a multiscale shift invariant wavelet decomposition is used. This Euclidean function will reduce the noise. Real and computer generated images are tested with this technique. The algorithm is analyzed using Figure of Merit, accuracy, sensitivity, specificity and false positive rate. In order to enhance the contrast of mammogram,a nonsampled contourlet transform is used. In this a multiscale non-separable edge filters are used to get the edge information depending on the hyperbolic tangent function. The overall contrast is improved by adaptive histogram equalization. Using this technique microcalcifications can be easily detected [12][13].

\section{METHODOLOGY}

Input retinal image is taken from DRIVE database and a sample image is shown in Fig 2 which is preprocessed for further processing. Preprocessing step includes noise removal, contrast enhancement and retinal boundary growth. Then the image is being processed in feature extraction model. The feature extraction model consists of Illumination Layer, Reflectance layer, Texture layer. Then, a minimum spanning superpixel tree detector is adopted to refine the segmentation results. The architecture of the proposed retinal recognition framework is shown in Fig 1 and it is discussed in this section. 


\section{Preprocessing Stage}
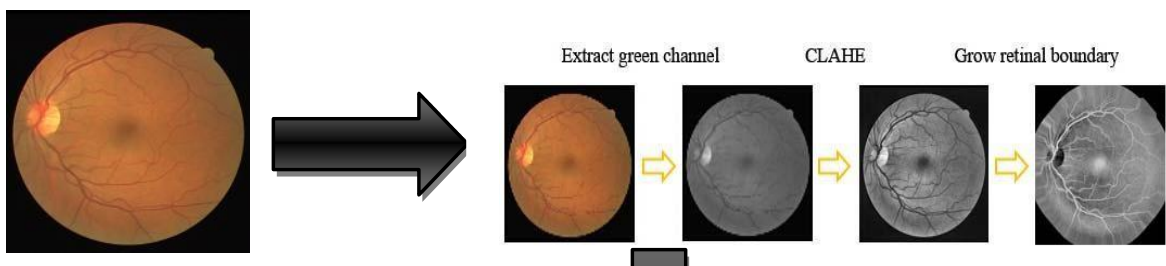

Input Image

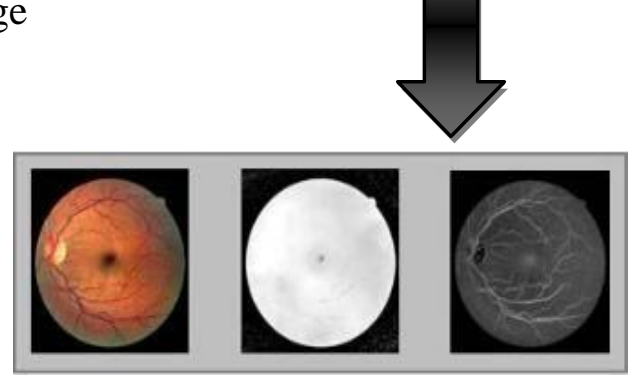

Vessel Segmentation

Feature Extraction

Fig 1. System architecture

In this work an simple linear iterative clustering (SLIC) algorithm is applied to get the superpixel of the retinal image by combining features obtained from distance measures based on spatial ,color and texture. The features are computed for two aims. First, superpixel features are obtained with the assumption that superpixel is the part of a vessel. Second, the input image gets divided into patches by the distribution of each pixel to the nearest pixel. A minimum spanning tree structure is constructed based on these superpixel texture, colour and space information, called Minimum Spanning superpixel Tree(MSST). Such MSST-based detector is employed to segment the retinal blood vessels. Finally, segmentation results are analysed. 


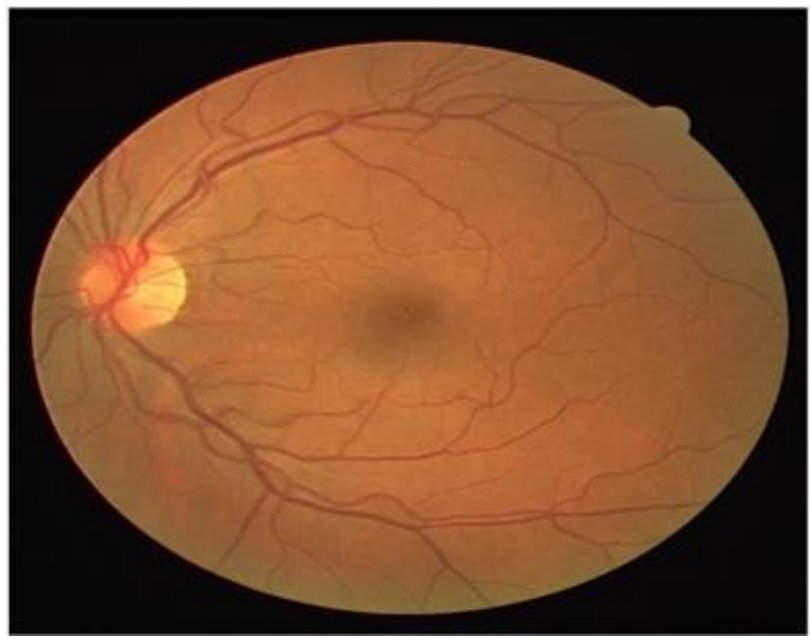

Fig 2: Input Retinal Image

\subsection{PRE-PROCESSING}

Fundus images have high contrast so, errors may occur in vessel detection around the edge area. A preprocessing is done for noise removal in such situation. Green channel image is separated as the blood vessel appears brighter in the green channel image and is shown in Fig 3. Contrast enhancement and retinal boundary growth is also done in this step for enhancing the segmentation task and is shown in Fig 4and Fig 5 respectively. The contrast limited adaptive histogram equalization (CLAHE) algorithm is employed to construct an image having local contrast enhancement. Here the image is divided into 64 areas ie, $8 * 8$. Inorder to remove the shortcomings from camera aperture border, a wavelet transformation is applied in combination with boundary germinating method involving a iterative method to amplify the specific area.

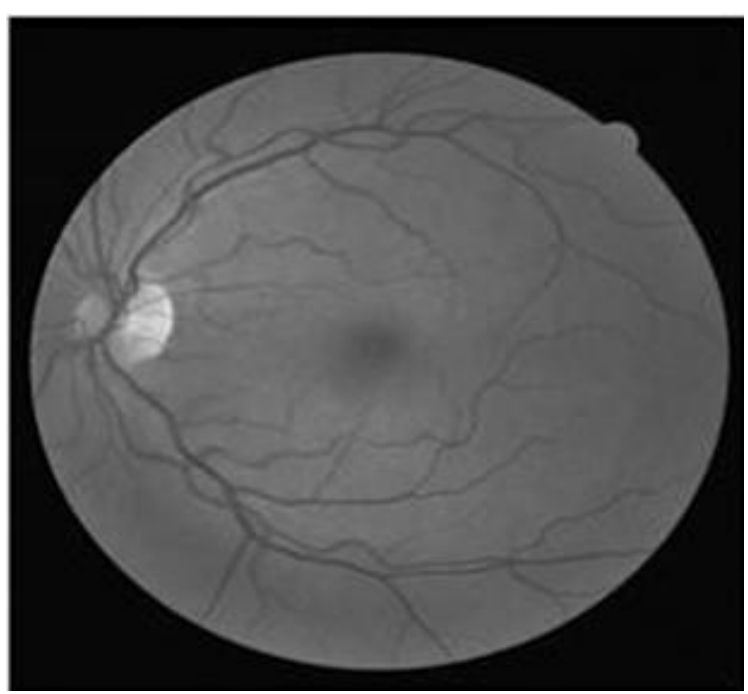

Fig 3 Green Channel Extracted Image 


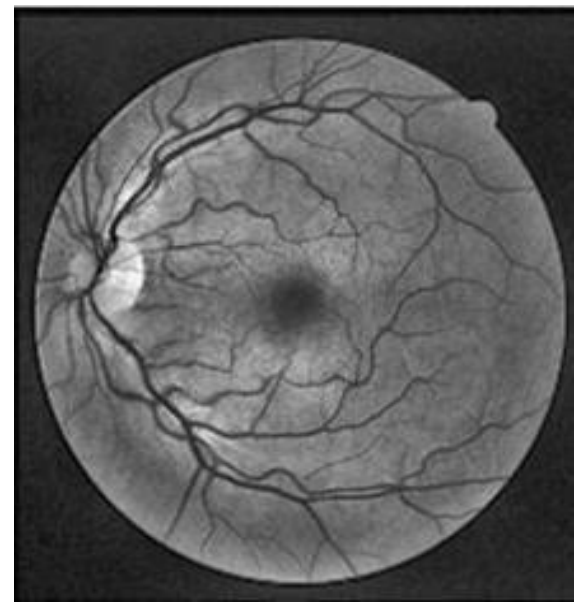

Fig 4 Contrast enhanced Image

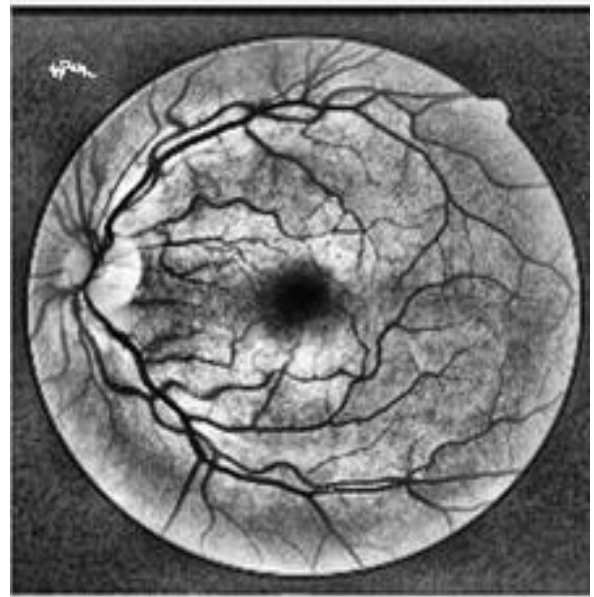

Fig 5 Retinal Boundary Image

\subsection{FEATURE EXTRACTION}

In feature extraction information is obtained from two layers as explained below.

\subsubsection{Illumination Layer}

From the anatomical studies it is understood that illumination layer is smoother than reflectance layer. The illumination layer is taken as L1, reflectance layer is taken as $L 2$, The image model is defined as

$$
I=\mathrm{L} 1+L 2
$$

An gradient is practised on reconstructed layers and an constraint other than this on the illumination layer. To obtain likeness with original image,a probability-based method is applied.The reflectance layer is not at all dim, still no proper illumination and scattering in the field of view, for few regions.

\subsubsection{Texture Layer}

Since textures provide spatial color and intensity information, it is chosen for superpixel-based vessel segmentation. Since, Gabor wavelets have orientation selectivity, multiscale properties, it is applied for texture analyzing. Gabor transform is defined as:

$$
\mathrm{T}_{\varnothing}(\mathrm{b} 1, \theta 1, \mathrm{a} 1)=\mathrm{c}_{\varnothing}^{\frac{-1}{2}} \mathrm{a} 1^{-1} \int \emptyset^{*}\left(\mathrm{a} 1^{-1} \mathrm{r}_{-\theta 1}(\mathrm{x} 1-\mathrm{b} 1)\right) \mathrm{I}(\mathrm{x} 1) \mathrm{d}_{\mathrm{x} 1}^{2}
$$

To obtain a good vessel segmentation ,the $\theta$ is limited from 0 to 170 . The maximum wavelet feedback for each and every pixel is,

$$
\mathrm{M}_{\varnothing}(\mathrm{a} 1, \mathrm{~b} 1)=\max \left|\mathrm{T}_{\varnothing}(\mathrm{b} 1, \theta 1, \mathrm{a} 1)\right|
$$

Since the blood vessels width fluctuates between $50 \mu \mathrm{m}$ and $200 \mu \mathrm{m}$, and the median value is also $60 \mu \mathrm{m}$, the value of a1 is set to 1.5. The superpixel is framed by using both the information of illumination layer and texture layer. This will help to generate superpixels 
efficiently.

\subsection{MINIMUM SPANNING SUPERPIXEL TREE DETECTOR}

In segmentation task a new approach called minimum spanning superpixel tree detector is applied which is explained as follows.

Superpixels are framed by features obtained from the illumination and texture layer and employing these features to the SLIC algorithm .This will split the original image as a assembly of superpixels. SLIC starts the sampling task with a spacing of $S=\sqrt{N} / \mathrm{K}$ pixels apart, from the cluster centers in a regular manner. To avoid the possibility of having the cluster center at the edges it is moved to the seed at the lowest gradient in a 3 by 3 neighborhood. Then, the clustering is done in an iterative manner to each and every pixel in the nearest cluster center. Finally, pixel connectedness is enriched by again labelling the separate segments from the biggest neighboring cluster labels. SLIC is much easier to operate and the boundary can be detected easily. Since, SLIC needs only one parameter to set the number of superpixels. SLIC clusters image pixels, using a weighted distance metric using color, texture, and image planes. Both in illumination and texture layer ,the distance between a pixel and the SLIC cluster center Ck is calculated. The spatial distance must be taken into account since the pixel position $[\mathrm{x}, \mathrm{y}] \mathrm{T}$ varies if the image size varies. If $6 \mathrm{D}$ Euclidean distance is employed it will cause difficulty in clustering superpixels ,if they are of varied sizes. For large superpixels, spatial distance measure will be greater than illumination measure. More importance is given to the spatial than to color. Such technique will frame superpixels that do not duplicate at edges. But in smaller superpixels, results will be more precise. The distance measure $\mathrm{D}$ is given as :

$$
\begin{gathered}
d_{a}=\sqrt{\left(R_{1}-R_{2}\right)^{2}+\left(G_{1}-G_{2}\right)^{2}+\left(B_{1}-B_{2}\right)^{2}} \\
d_{t}=\sqrt{\left(g_{1}-g_{2}\right)^{2}} \\
d_{s}=\sqrt{\left(x_{1}-x_{2}\right)^{2}+\left(y_{1}-y_{2}\right)^{2}} \\
D=\sqrt{\left(m_{a} d_{a}^{2}+m_{t} d_{t}^{2} m_{s} d_{s}^{2}\right.}
\end{gathered}
$$

where R,G,B denote the red, green, and blue channels of the illumination layer, the pixel position, and the coordinates of the cluster center respectively. $d_{a}, d_{t}$ and $d_{s}$ represent the illumination, texture, and spatial closeness respectively and $m_{a}, m_{t}$ and $m_{s}$ are the weights of the illumination, texture, and spatial distance measures respectively.

By setting the weight value equal to the difference between pixel and the cluster center, the features of both illumination and texture is taken into account. Since the information of three layers are combined, Superpixel blocks adhere well to vessel boundaries. By clustering pixels into superpixels, illumination noise is removed from fundus images. Every vessels both big and small vessels are enhanced, because inherent homogeneities are spread to their neighbors by k-means clustering. When superpixels is framed, the superpixel attributes will be a set of local similar pixels. Vessel superpixels will be edge areas, depending on an 
averaging operator which smoothens the small homogenous vessel regions. They donot have original sharp edges, since geometric image structures are ignored. The retinal image which is formed into superpixels is given in Fig 6.

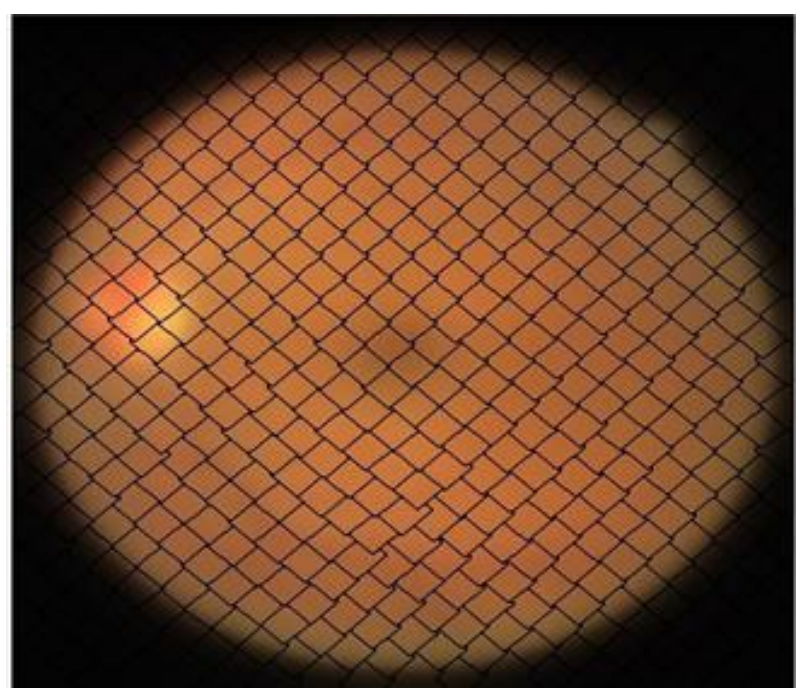

Fig 6. Superpixel Image

A new edge preserving tree detector is employed to evaluate the vesselness of superpixels, by determining weighted average. The tree detector distinguishes small connected components from large connected components by using a minimum spanning tree, to handle connected components in superpixel. An MSST is formed by eliminating edges having larger weights, such that two unlike superpixels are spontaneously pulled apart, seed point in a manual manner. The MSST method will not loose the information but has the capability to store the informations in the margins of vessels. The minimum weight is searched to get the global features. It has low complexity. Since retinal vessels are like vascular tree structures, we associate the vessel superpixel sets by constructing the MSST. By using the proposed MSST ,accurate segmentation is achieved in minimum time. An MSST is constructed by using Kruskals algorithm where a superpixel graph is treated as a standard 4-connected grid whose nodes are the partitioned superpixels.

\section{RESULT AND DISCUSSION}

The input image is taken from the DRIVE database. A sample image from the database is resized into 215 x 215 size. In pre-processing stage, Green channel image is separated followed by contrast limited adaptive histogram equalization (CLAHE) algorithm to get local contrast enhancement. Next a boundary germinating method is applied. The illumination, reflectance and texture layer are obtained. The illumination layer is used for superpixel based segmentation is shown in Fig 7. 


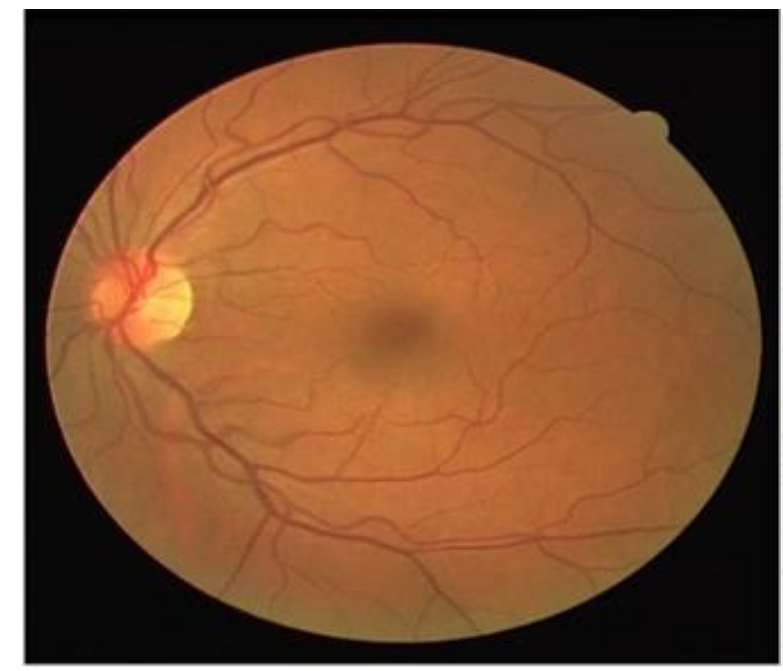

Fig 7. Illumination Layer

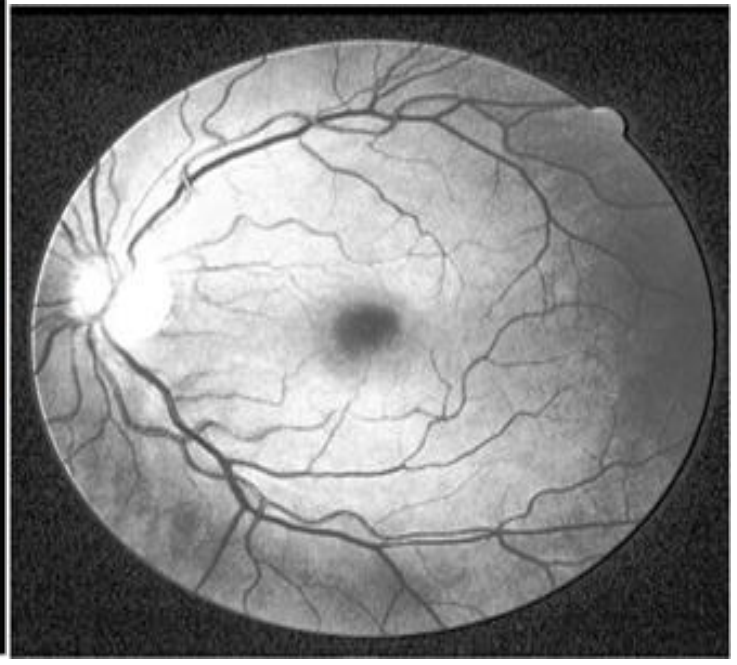

Fig 8. Reflectance Layer

The reflectance layer produces very bright vessel patterns. The vessel patterns are shown in Fig 8. Gabor wavelets used in texture layer detectors, for texture analyzing. The texture layer is shown in Fig 9.

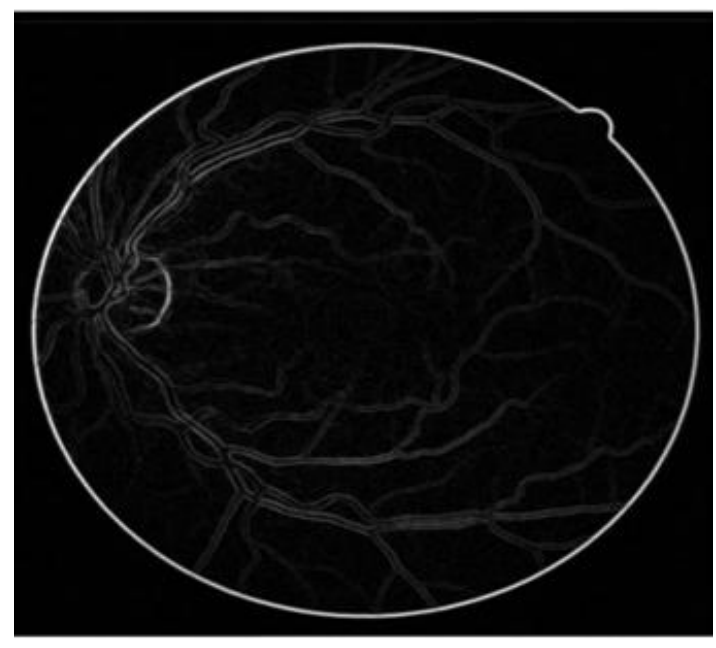

Fig 9. Texture Layer

Finally MSST is used to segment the vessel part effectively. The vessel segmentation is shown in Fig 10. 


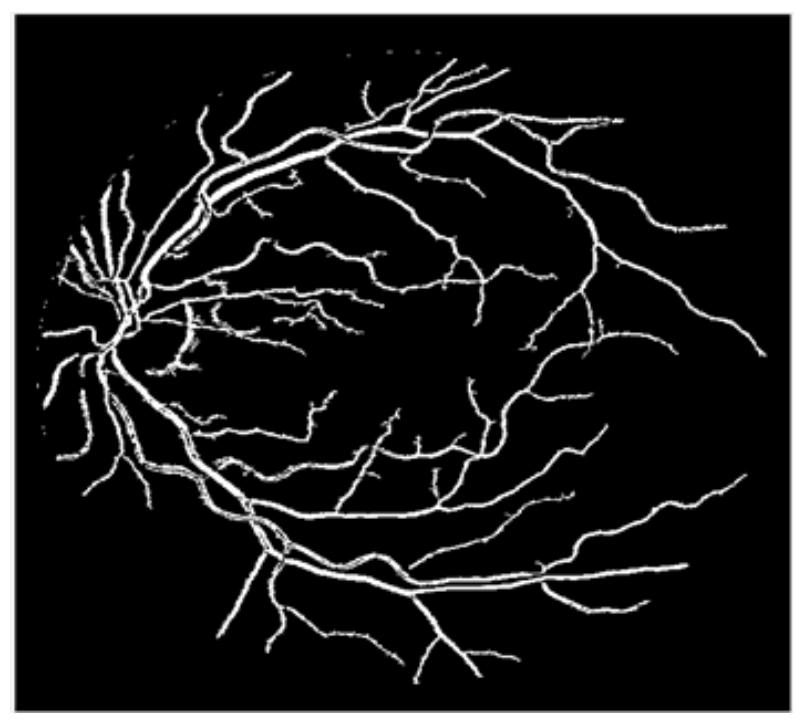

Fig 10. Blood Vessel Segmentation

Table 1 shows the performance of the proposed method with few existing methods like levelset, watershed transform etc.

Table. 1 Performance of different segmentation models

\begin{tabular}{|l|l|l|l|l|}
\hline Method & Accuracy & Sensitivity & Specificity & Time \\
\hline Level set & 94.5 & 91 & 96 & 7.50 \\
\hline $\begin{array}{l}\text { Watershed } \\
\text { Transform }\end{array}$ & 95.1 & 92 & 95 & 7.25 \\
\hline $\begin{array}{l}\text { Proposed } \\
\text { method }\end{array}$ & 97.6 & 91 & 95 & 8.50 \\
\hline
\end{tabular}




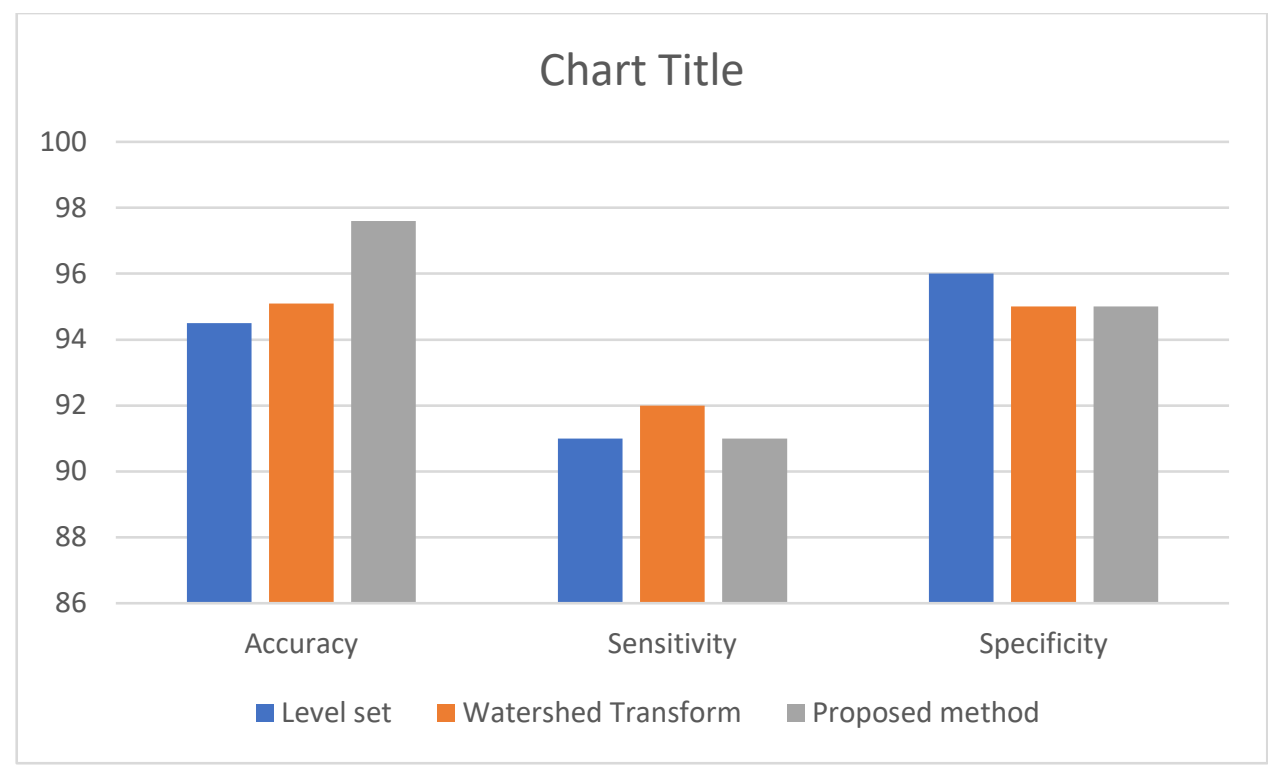

Fig 11. Performance chart

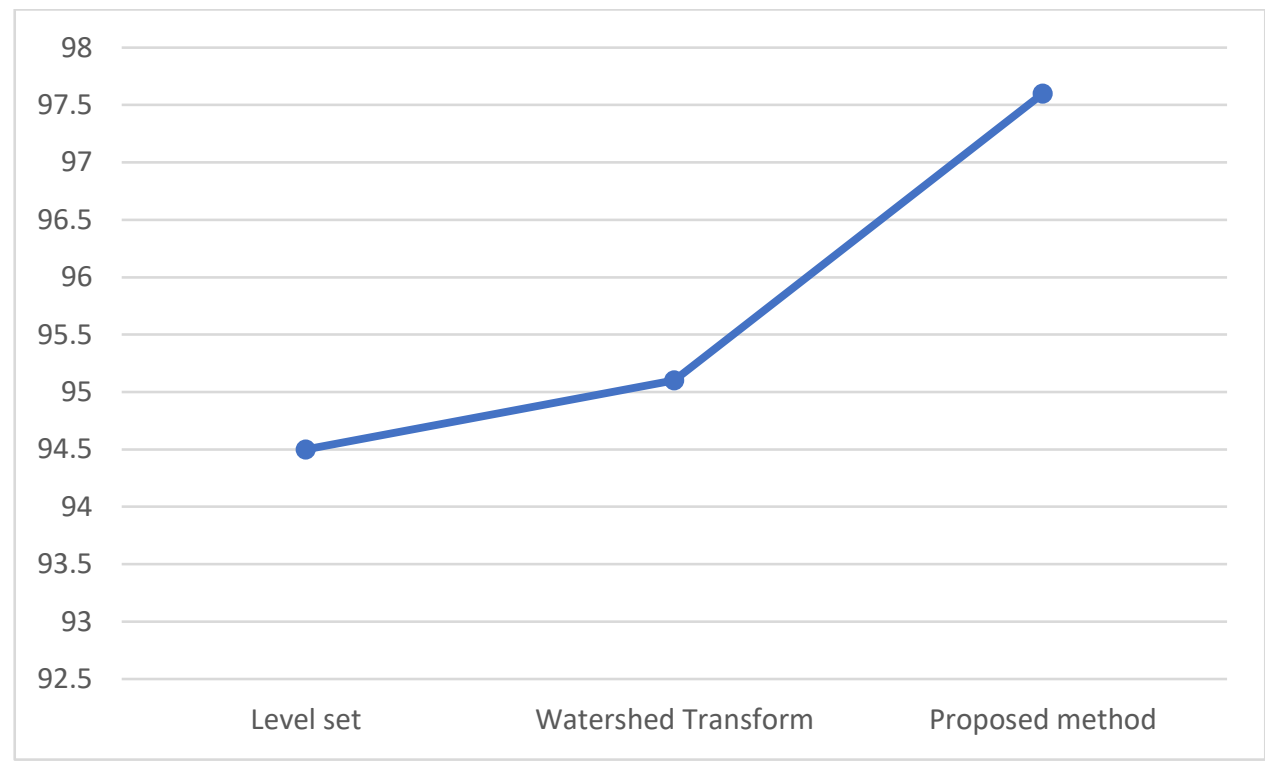

Fig 12. Time of execution chart

The performance is analysed in terms of accuracy, sensitivity, specificity and time of execution. It is found that the proposed method gives an accuracy of 97.6 and also it executes faster with a computation time of $8.50 \mathrm{sec}$. Fig 11. shows the pictorical variations of performance of the proposed method. From Fig 12. it is understood graphically that the proposed method executes faster than other techniques.

\section{CONCLUSION}

Effective vessel segmentation for retinal images is an important area in medical imaging. A new efficient method of superpixel based tree structure is employed for retinal vessel segmentation. In the proposed method, a combination of global shape, texture, color, 
and space information is used, instead pixel information. The experimental result shows that it provide high accuracy of $97.6 \%$ and executes within $8.50 \mathrm{sec}$. Future work will be to continue to study the characteristics of image methods to increase Sensitivity, specificity and Accuracy value with various other databases.

\section{REFERENCES}

[1] Chaudhuri, S., Chatterjee, S., Katz, N., Nelson, M., \& Goldbaum, M. (1989). Detection of blood vessels in retinal images using two-dimensional matched filters. IEEE Transactions on medical imaging, 8(3), 263-269.

[2] Wang, X., \& Jiang, X. (2017, March). Enhancing retinal vessel segmentation by color fusion. In 2017 IEEE International Conference on Acoustics, Speech and Signal Processing (ICASSP) (pp. 891895). IEEE.

[3] Nguyen, U. T., Bhuiyan, A., Park, L. A., \& Ramamohanarao, K. (2013). An effective retinal blood vessel segmentation method using multi-scale line detection. Pattern recognition, 46(3), 703-715.

[4] Ricci, E., \& Perfetti, R. (2007). Retinal blood vessel segmentation using line operators and support vector classification. IEEE transactions on medical imaging, 26(10), 1357-1365.

[5] Al-Rawi, M., Qutaishat, M., \& Arrar, M. (2007). An improved matched filter for blood vessel detection of digital retinal images. Computers in biology and medicine, 37(2), 262-267.

[6] Fraz, M. M., Remagnino, P., Hoppe, A., Uyyanonvara, B., Rudnicka, A. R., Owen, C. G., \& Barman, S. A. (2012). An ensemble classification-based approach applied to retinal blood vessel segmentation. IEEE Transactions on Biomedical Engineering, 59(9), 2538-2548.

[7] OSAREH, A., \& Shadgar, B. (2009). Automatic blood vessel segmentation in color images of retina. Iranian Journal of Science and Technology Transaction B- Engineering, 33,191-206.

[8] Cheng, E., Du, L., Wu, Y., Zhu, Y. J., Megalooikonomou, V., \& Ling, H. (2014). Discriminative vessel segmentation in retinal images by fusing context-aware hybrid features. Machine vision and applications, 25(7), 1779-1792.

[9] Abhisha Mano \& Anand, S.(2019). GSO based weight Optimized Artificial Neural Network with Modified Active Contour for Brain Tumor Segmentation. Tierärztliche Praxis,39(11).184-204.

[10] Premkumar, R., \& Anand, S. (2019). Secured and compound 3-D chaos image encryption using hybrid mutation and crossover operator. Multimedia Tools and Applications, 78(8), 9577-9593.

[11] Anand, S., Lakshmanan, B., Murugachandravel, J., Valarmathi, K., Mano, A., \& Kavitha, N. (2019).Wavelet-Based Automated DNA Sizing of Fragments through AFM Image Processing. International Journal of Engineering and Advanced Technology.8(5) .231-238.

[12] Anand, S., Murugachandravel, J., Valarmathi, K., Abhisha Mano \& Kavitha,N.(2019). Contrast Enhancement of Mammograms and Microcalcification Detection. International Journal of Recent Technology and Engineering (IJRTE) ,8(4).3926-3932.

[13] Anand, S., \& Sindhuja, N. M. (2009, June). Spot edge detection in microarray images using balanced GHM multiwavelet. In 2009 International Conference on Control, Automation, Communication and Energy Conservation (pp. 1-4). IEEE. 
